\title{
AERO-OPTICAL CHARACTERISTICS OF COMPRESSIBLE, SUBSONIC TURBULENT BOUNDARY LAYERS
}

\author{
Stanislav Gordeyev* and Eric J. Jumper** \\ Center for Flow Physics and Control \\ Department of Aerospace and Mechanical Engineering \\ The Hessert Laboratory \\ University of Notre Dame \\ Notre Dame, Indiana \\ T. Terry $\mathrm{Ng}^{\dagger}$ and Alan B. Cain ${ }^{\dagger \dagger}$ \\ Innovative Technology Applications Company \\ Chesterfield, Missouri
}

\begin{abstract}
An extensive experimental study of optical aberrations due to propagation through fully-developed turbulent boundary layers at high subsonic Mach numbers was performed. Time-resolved, highbandwidth, direct optical measurements of the dynamic aberrations were made using a Malley probe. The probe was used to obtain the convective speeds of the optically-significant turbulence structures and to measure the optical path differences. Measurements were made over a range of boundary layer thicknesses and Mach numbers. Optical distortions were found to scale linearly with boundary layer thickness and freestream density, and to go as the square of the freestream Mach number.
\end{abstract}

\section{INTRODUCTION}

When an otherwise-planar, optical wavefront is made to propagate through a variable-index-ofrefraction, relatively-thin, turbulent flow, the wavefront becomes aberrated. The study of the effect of optical propagation through such flow fields is referred to as aero-optics. ${ }^{1,2}$ The rapidly-time-varying aberrations

* Member AIAA, Assistant Research Professor.

**Fellow AIAA, Professor.

† Senior Member AIAA, Vice-President.

It Associate Fellow AIAA, President

Copyright (๑ 2003 by S. Gordeyev, E. Jumper, T. Ng and A. Cain Published by the American Institute of Aeronautics and Astronautics, Inc. with permission. imposed on the wavefront degrade the performance of an optical system attempting to make use of the optical signal. Variable-index turbulent flow fields may have their origin in the mixing layer between two dissimilarindex flow streams (two-index mixing), ${ }^{3,4}$ or may be caused by projecting the optical beam through highMach, boundary and free shear layers. The latter scenarios are relevant to the use of lasers on flight vehicles with flight Mach numbers greater than 0.6., Propagation of laser beams through aero-optic flows are of interest to applications that range from weaponsrelated to free-space communication.

The optically-aberrating effects of high-speed, turbulent boundary layers have been the subject of research since the early 1950's, which produced the first theoretical formulation for the their effect based on statistical measures of the turbulence ${ }^{2}$. Work on the turbulent boundary layer intensified in the late 1960's and through the decade of the 1970's due to an interest in placing lasers on aircraft. Little was done on the effect of separated shear layers until the 1990's; however, as it turns out, much more is known today on the optical effects of separated shear layers than on the older area of study, the turbulent boundary layer.

Here is what we know about the separated shear layer. For high-Mach-number, subsonic free shear layers, the associated flow fields are generally considered "incompressible." For a long time, it was thought that these layers should be relatively benign to optical propagation; yet, since the early 1990's, it has been shown that such layers greatly affect the beam quality (wavefront figure) of a laser propagated through 
them $^{7,8}$. Not only is it known that the shear layer has a large detrimental effect, but also known that it is the coherent structures that form (roll up) naturally in the layer that cause the aberration. In particular, it is the radial pressure gradients (in the convecting frame) required to support the curvature of the flow that cause pressure wells and their concomitant low densities, which, in turn, create index-of-refraction variations along and through the shear layer. ${ }^{9}$

For an attached turbulent boundary layer at high subsonic Mach numbers, the aberrating effect of propagation through the layer is less than that for propagation through a free shear layer; however, even though the optical effects of turbulent boundary layers have been studied since the early 1950's, unlike the free shear layers, the physical causes of the aberrations are not known, other than that they are caused by the fluctuating density.

Until the present study, Rose ${ }^{10}$ performed the most extensive experimental studies of optical aberrations caused by a turbulent boundary layer. He conducted hot-wire measurements in turbulent boundary layers in order to obtain their density fluctuations, $\rho^{\prime}(\mathrm{y})$, and associated correlation lengths, $\Lambda_{\rho}(\mathrm{y})$. These were used to estimate wavefront aberrations that would be imprinted on a laser beam propagated through the same turbulent boundary layer. The on-average wavefront aberrations, in the form of $\mathrm{OPD}_{\mathrm{rms}}$ (Optical Path Difference), were estimated using a now-well-known integral "linking equation;",2,3 some of Rose's measurements were performed on an in-flight aircraft's turbulent boundary layer. Rose ${ }^{10}$ empirically found $\mathrm{OPD}_{\text {rms }}$ is proportional to a dynamic pressure and a boundary layer thickness. The aircraft hot-wire measurements were complemented by the work of Gilbert $^{5}$, who performed interferometer measurements. In the Gilbert work, the interferometry used a doublepulse technique, which actually measured the difference in the wavefront from one pulse to another, rather than the distorted wavefront at a given instant, and only a limited number of these were made. Gilbert reported that the interferometry generally supported the hotwire, integral-method estimations of the $\mathrm{OPD}_{\text {rms. }}$ In 1994, Masson et. al. ${ }^{6}$ revisited the Gilbert and Rose data and concluded that while the trends between the two techniques of estimating the optical aberrations appeared to be generally the same, there was what appeared to be a systematic difference in the arrived upon values, with the interferometric estimates consistently yielding higher estimates of the $\mathrm{OPD}_{\mathrm{rms}}$ than the hot-wire estimates. These trends were reported by Gilbert as the square of the $\mathrm{OPD}_{\mathrm{rms}}$ depended linearly on the dynamic pressure.
Given the relatively-large body of information already in the literature on the optically-aberrating effects of turbulent boundary layers, one might reasonably ask the question why still one more paper on this topic is needed ${ }^{2}$. To start with, Masson's paper ${ }^{6}$, which appeared many years after the research activities for the turbulent boundary layers, raises a question about the accuracy of the measurements (fluidmechanic and optical) previously reported. But beyond this, there have been a number of developments since the earlier boundary-layer, aero-optical work. First, the magnitude of the $\mathrm{OPD}_{\mathrm{rms}}$ predicted from the earlier work was considered inconsequential at the time because its application was for an airborne gasdynamic laser $\left(\mathrm{CO}_{2}\right)$ that lased at $10.6 \mu \mathrm{m}$. The system impact of these aberrations can be assessed in a number of ways; however, one of the most common is to quantify them in terms of a time-averaged Strehl ratio, defined as

$$
\overline{S_{t}}=\frac{\bar{I}}{I_{o}}
$$

where, $I_{o}$ is the distortion-free (diffraction-limited) value of the intensity on the target and $\bar{I}$ is actual, average value of the intensity. The $\mathrm{OPD}_{\text {rms }}$ can be used to estimate the Strehl ratio using the "large aperture approximation," given by ${ }^{11}$

$$
\overline{S_{t}}=\exp \left[-\left(\frac{2 \pi O P D_{r m s}}{\lambda}\right)^{2}\right]
$$

Notice that the exponent scales as $(1 / \lambda)^{2}$; today, wavelengths of interest are in the near-IR $(\sim 1 \mu \mathrm{m})$ and visible. Thus, the $\mathrm{OPD}_{\text {rms }}$ predicted by Gilbert and Rose that were known to be inconsequential $(\sim 0.1 \mu \mathrm{m})$ at $10.6 \mu \mathrm{m}$ now reduce the Strehl ratio by about $30 \%$, and, given Masson's observations and other concerns about the accuracy of estimating the $\mathrm{OPD}_{\text {rms }}$ using the earlier methods, there is now a renewed interest in revisiting the turbulent-boundary-layer problem.

Secondly, there now exists direct optical instrumentation capable of assessing not only the $\mathrm{OPD}_{\text {rms }}$, but also the spatial and temporal frequencies of the aberrations, and, as will be described below, the ability to construct wavefronts from which the far-field intensity pattern can be computed directly. Using the time-resolved time series of far field patterns, Strehl ratio as a function of time can be computed. From the point of view of applications to free-space communication, which are concerned with bit error rate, these time series of instantaneous Strehl ratio are more instructive than their average; however, once the time series are available the time-averaged Strehl ratio 
can be computed, which is of interest to weapons applications.

Thirdly and finally, because conformal windows (which presume an attached turbulent boundary layer), as opposed to beam-directing turrets (which require propagation through regions of separated flow), are now being considered for exit apertures, it is critical that a more reliable method of predicting the turbulentboundary-layer's aberration is needed. Not only is it important to measure the aero-optical effects of the turbulent boundary layer for propagation normal to the boundary layer, it is now possible, and imperative to measure its effects at all relevant slant angles. In addition, with the new ability to obtain direct, highfidelity optical data without resorting to indirect methods of predicting the optical aberrations, it is now more efficient to revisit the question of scaling of the aero-optic data.

With this in mind, the purpose of this paper is to present a new study of the aero-optical effects of propagation through a turbulent boundary layer over a range of boundary-layer thicknesses and free stream Mach number ${ }^{5}$. This paper presents results only for normal propagation through the layer; however, these data are used to as a basis for a scaling law that allows for the extending the results to other Mach numbers, boundary layer thicknesses and altitudes.

\section{EXPERIMENTAL SET-UP}

The experiments reported on here were performed in one of three transonic indraft wind tunnels in the Hessert Laboratory at the University of Notre Dame. The tunnel used consists of a 150:1 contraction inlet, a test section, and a diffuser (see Fig. 1). The diffuser joins to a large gated plenum, which is common to all three tunnels. The plenum is pumped to low pressure by up to three Allis Chalmer 3,310 CFM vacuum pumps. By selecting the proper valve settings, the tunnel can be pumped by one, two or three pumps; the present experiments used either one or two pumps depending on the specific Mach number.

The test section, whose cross section is $9.9 \mathrm{X} 10.1$ $\mathrm{cm}$, could be reconfigured to allow for the section to be lengthened or shortened, adjusting the boundary layer thickness. In addition, the lower side of the test section (which was the side of the tunnel whose boundary layer we investigated) was covered with a medium grain sandpaper along its first $50 \mathrm{~cm}$ to facilitate the onset and initial growth of a turbulent boundary layer. The boundary layer was then allowed to grow naturally from that point to a tunnel length of from 60 to $110 \mathrm{~cm}$, depending on the test section's configuration. The flow then entered the measurement portion of the test section, shown in Figure 2. The measurement section is made from Plexiglas and instrumented with both total and static pressure ports to monitor the flow speed. Although Plexiglas can hardly be considered to be of "optical quality," it is transparent and of sufficient quality for measuring the optical aberrations using a Malley probe, which will be described in more detail below. A first-surface mirrored optical flat was mounted at the mid-height of the measurement section on a specially-shaped mounting plate, that assured no separated flow over the mirror's span. The upper wall of the measurement section was contoured to compensate for a blockage effect of the mirror mounting plate. The mirror served a dual purpose: it

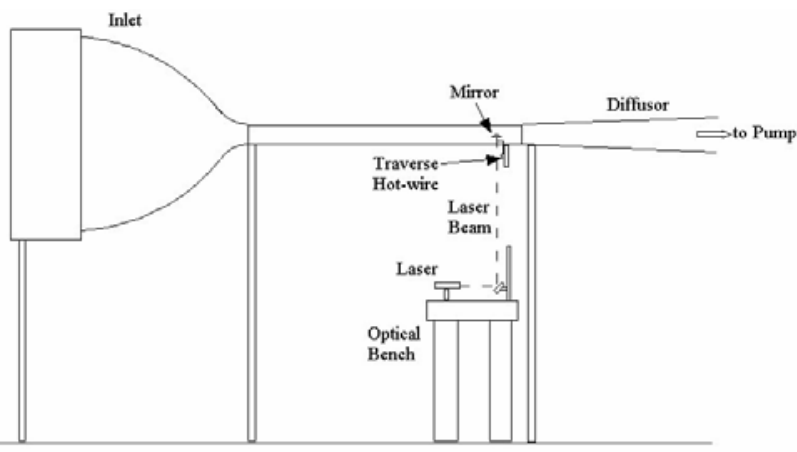

Figure 1. The schematic of the boundary layer facility.

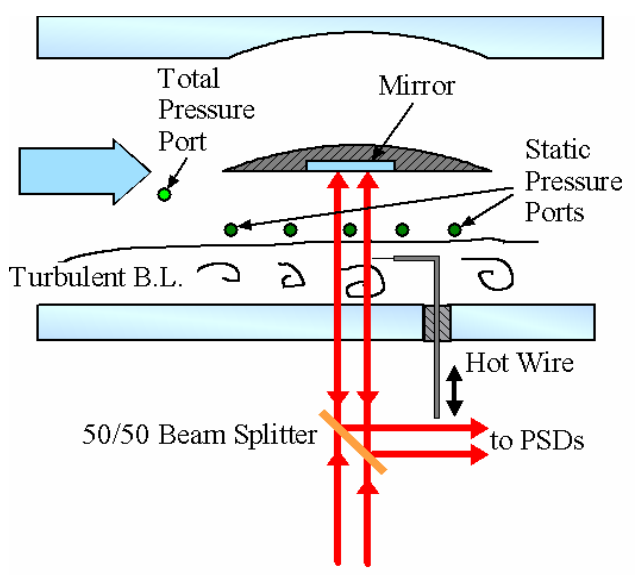

Figure 2. Measurement station instrumentation.

eliminated the complications that would have been present due to propagation through both the lower-wall boundary layer and the upper-wall boundary layer; and, by reflecting the measurement beams directly back along their propagation path back through the same 
boundary layer at its same location, it doubled the optical path through the bottom boundary layer, increasing the measurement signal-to-noise ratio, without ambiguity. The range of the Mach numbers investigated was between $M=0.3$ and 0.95 .

In the following sections, the streamwise direction, $x$, starts from zero at the beginning of the duct/nozzle interface, extending from zero there, over the roughened surface to the length at which the measurement is made, which will be reported as the value $L$. The vertical propagation distance, $y$, starts from zero at the lower wall.

Malley Probe. A Malley probe was used to perform the optical measurements. The Malley Probe is an optical instrument that can make direct, accurate measurements of dynamically-distorting wavefronts, including the characteristics of the $\operatorname{OPD}(t)$. By moving the instrument, an entire, large aperture can be optically characterized. This characterization includes not only the measurement of $\mathrm{OPD}_{\mathrm{rms}}$, but also the spatial and temporal frequencies of the aberrations. The instrument itself is a further development by Notre Dame of an instrument described in a paper by Malley, et. al. ${ }^{12}$. In that paper, a working instrument was developed and applied to an aero-optical flow and shown to be consistent with $\mathrm{OPD}_{\text {rms }}$ estimates made using a limited number of interferograms for the same flow field. The Notre Dame, Malley-derivative sensor is an advance over the one described in Malley, et. al., and has now been shown to give extremely accurate measurements of $\mathrm{OPD}_{\mathrm{rms}}{ }^{13}$. The instrument consists of two closely spaced beams ( 3-8 $\mathrm{mm}$ apart and aligned, front beam to aft beam, in the streamwise direction); the second beam is used to extract phase-velocity data contained on the beam-deflection angles by cross correlating them and obtaining the time delay for maximum correlation. Knowing the displacement between the beams and this delay time, the phase velocity can be computed. As described in Hugo and Jumper ${ }^{13}$, the deflection angle of the probe beam is the spatial derivative of a wavefront for a larger-aperture, otherwise planar wavefront that would be present if that wavefront were aberrated by the same flow. The group velocity is needed to unfold the OPD using the fact that the aberrations "convect" with convecting fluid structures; this fact was first discussed by Malley et. al. ${ }^{12}$ A schematic and a photograph of the actual set-up for these experiments are shown in Fig. 3.

The beam separation was set at $4.2 \mathrm{~mm}$ throughout all measurements. After traversing the test section and passing through the applicable lens, the beams were directed to two positions sensing devices, PSD, the signal from the PSD's was used to record the displacement of the beams as a function of time, from which the wavefront slope could be determined. Based on preliminary measurements of the temporal frequencies present in the dynamic aberrations, the sampling rate was set to $\mathrm{f}_{\text {samp }}=500 \mathrm{kHz}$. Total number of points per channel was 16384, giving a total sampling time of $32.8 \mathrm{msec}$. Two test section lengths, $L=110$ and $160 \mathrm{~cm}$, and four Mach numbers, $M=0.3$, $0.5,0.7$ and 0.9 , were used for the data presented here.
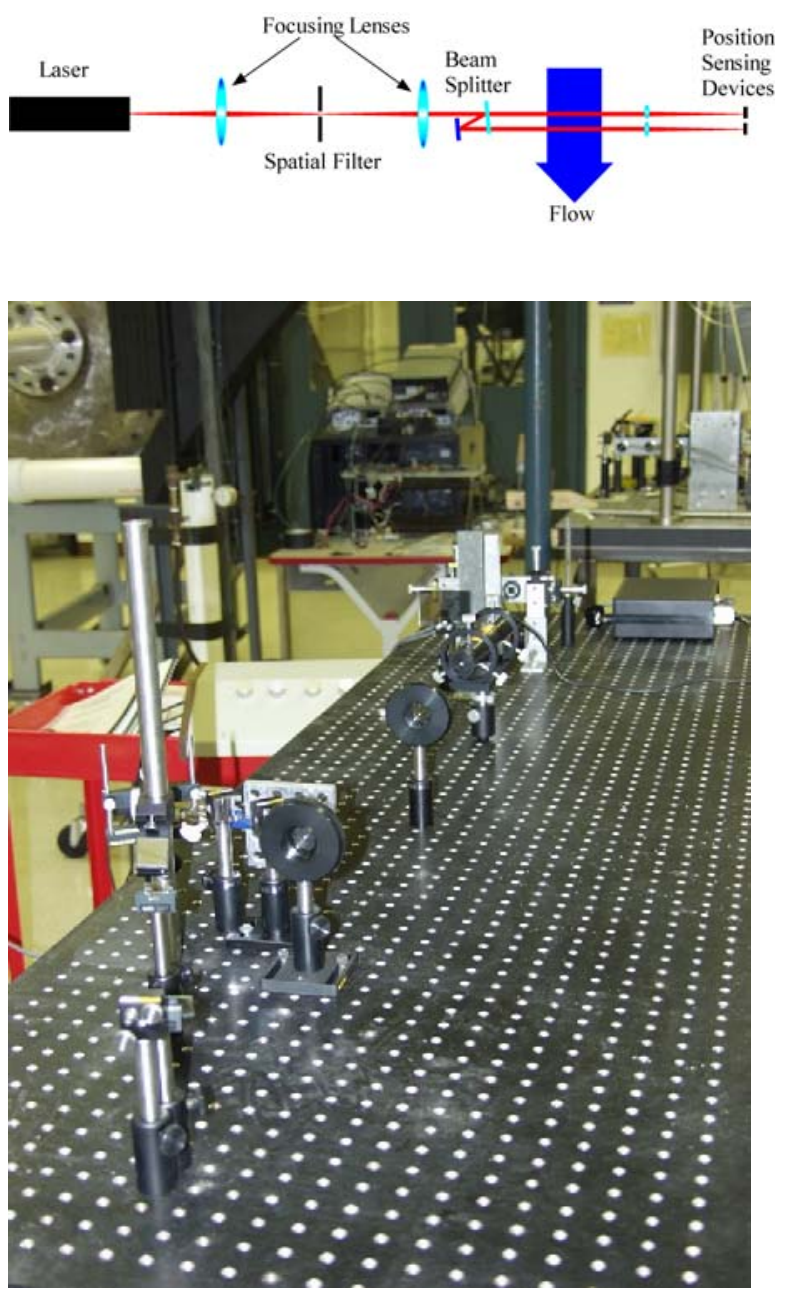

Figure 3. A Malley probe: schematic and actual setup.

Hot-Wire Measurements. In conjunction with the optical measurements, conventional hot-wire measurements were performed to obtain boundary layer profiles. A single hot-wire was used with a commercial constant temperature anemometer with a built-in lowpass filter. The sampling rate for hot-wire measurements was $60 \mathrm{kHz}$, with a low-pass frequency cut-off value at $30 \mathrm{kHz}$. The hot-wire was placed inside 
the measurement section on a traverse system just behind the optical measurement location. The hot-wire was calibrated at the range of Mach numbers from $M=$ 0.2 to 0.6. Boundary layer profiles $U(y)$ and $u_{\mathrm{rms}}(y)$ were taken for two test sections length at $M=0.3$ and 0.5 and the displacement thicknesses

$$
\delta^{*}=\int_{0}^{\infty}\left(1-U(y) / U_{\infty}\right) d y
$$

were calculated.

The flow inside the tunnel was assumed to be isentropic and adiabatic in the core flow, from which local density, $\rho$, temperature, $T$, and speed of sound, $a$, were calculated as functions of $M$.

Aberration Convective Speed Determination. As mentioned in the previous section, the Malley probe directly measures a convective speed of aberrations by computing a time-delayed correlation function

$$
R(\tau)=\overline{\theta_{1}(t) \theta_{2}(t+\tau)}
$$

between two signals $\theta_{1}(t)$ and $\theta_{2}(t)$ from the two probe beams. A convective speed $U_{c}$ is computed knowing a separation $s$ between beams and a time delay of the highest correlation, $\tau^{\max }, R\left(\tau^{\max }\right)=\max$, that is

$$
U_{c}=\mathrm{s} / \tau^{\max }
$$

Another way of computing the time delay is to analyze a spectral cross-correlation function

$$
S(\omega)=\int R(\tau) \exp (-i \omega \tau) d \tau
$$

It can also be rewritten in terms of Fourier transforms of the signals,

$$
S(\omega)=\frac{1}{T}\left\langle\hat{\theta}_{1}(\omega) \hat{\theta}_{2}^{*}(\omega)\right\rangle
$$

where the hat denotes a Fourier transform, the asterisk denotes a complex conjugate, $T$ is a block sampling time and the brackets denote an ensemble average. Assuming a non-changing convecting structure without any noise, the signal downstream, $\theta_{2}$, is just a timedelayed signal of the upstream signal, $\theta_{1}$,

$$
\theta_{2}(t)=\theta_{1}\left(t-\tau^{\max }\right)
$$

The Fourier transform of $\theta_{2}$ becomes

$$
\begin{aligned}
& \hat{\theta}_{2}(\omega)=\int \theta_{2}(t) \exp (-i \omega t) d t= \\
& \int \theta_{1}\left(t-\tau^{\max }\right) \exp \left(-i \omega\left[t-\tau^{\max }\right]\right) \exp \left(-i \omega \tau^{\max }\right) d t
\end{aligned}
$$

$$
=\hat{\theta}_{1}(\omega) \exp \left(-i \omega \tau^{\max }\right)
$$

Using this relation and computing the spectral correlation $S(\omega)$,

$$
\begin{aligned}
& S(\omega)=1 / T\left\langle\hat{\theta}_{1}(\omega) \hat{\theta}_{2}^{*}(\omega)\right\rangle \\
& =1 / T\left\langle\hat{\theta}_{1}(\omega)\left[\hat{\theta}_{1}(\omega) \exp \left(-i \omega \tau^{\max }\right)\right]^{*}\right\rangle \\
& =A(\omega) \exp \left(i \omega \tau^{\max }\right)
\end{aligned}
$$

where $A(\omega)=1 / T\left\langle\hat{\theta}_{1}(\omega) \hat{\theta}_{1}^{*}(\omega)\right\rangle$ is a real function of $\omega$. Thus, by analyzing the argument of the spectral cross-correlation function, $S(\omega)$, one can find the time delay by a least-square estimation,

$$
\left|\exp \left(i \operatorname{Arg}[S(\omega)]-i \omega \tau^{\max }\right)\right|^{2} \rightarrow \min
$$

A complex exponent is introduced to eliminate phase ambiguity. In the results and discussion section it will be shown that the second method is more robust in computing the convection velocity than the Eq. (5) method when low-frequency noise is present in the signals; low frequency noise is always present due to tunnel vibration.

Optical Path Difference (OPD) Construction. The PDS's measure locations of the beams' centroids at lenses' focal points as a function of time. The PSD's response times are on the order of 20 nanoseconds so that their signals have virtually no delay in their response; this allows high sampling rates and thus the ability to measure accurate correlations even with a close beam spacing. From the beam deflections, $\Delta(t)$, the angle of deflection $\theta(t)$ was calculated as

$$
\theta(t)=\frac{\Delta(t)}{f}
$$

where $f$ is the focal length of the focusing lenses ahead of the PSD's. A focal length, $f$, of $1.0 \mathrm{~m}$ was used in this experiment.

Optical Path Length (OPL) is related to the deflection angle by

$$
\theta(x, y, t)=\frac{d O P D(x, y, t)}{d x}
$$

Assuming a convective nature of structures, the spatial derivative can be replaced with the temporal one as

$$
\frac{d}{d x} \rightarrow-\frac{1}{U_{c}} \frac{d}{d t}
$$


where $U_{c}$ is the convective speed, measured using the Malley probe. So, the OPL can be computed from the temporal evolution of the deflection angle, $\theta(\mathrm{t})$, at the fixed location as,

$$
O P L(x) \approx-U_{c} \int_{t_{0}}^{t=x / U_{c}} \theta(t) d t
$$

In real applications only the relative variation of the OPL is of interest,

$$
O P D(x)=O P L(x)-\overline{O P L(x)}
$$

where the overbar denotes the average.

A second order quadrature integration procedure was used to numerically compute $O P D(x)$.

\section{RESULTS AND DISCUSSION}

Experimental results. Single hot-wire measurements were taken to obtain boundary layer velocity profiles. Figure 3 presents mean and fluctuating components of the streamwise component of the velocity at $L=160 \mathrm{~cm}$ downstream of the beginning of the test section. Analysis of the boundary layer profiles reveals the presence of a well-defined, log-law region in each case. It confirms that the turbulent boundary layer was fully developed by the time it entered the measurement section. The calculated displacement thicknesses as a function of the boundary layer growth length, $L$, and the core Mach number, $M$, are presented in Table 1.

Assuming a self-similarity of the turbulent boundary layer development is applicable,

$$
\delta^{*} \sim L /\left(\rho U_{\infty} / \mu\right)^{0.2}
$$

displacement thicknesses can be interpolated to other Mach numbers. Based on data from the Table 1, the best fit was found to be,

$$
\delta^{*}=11.7\left(\frac{L[m]}{1.6}\right)^{0.8}\left(\rho\left[\frac{\mathrm{kg}}{\mathrm{m}^{3}}\right] U_{\infty}\left[\frac{\mathrm{m}}{\mathrm{sec}}\right]\right)^{-0.2}(\mathrm{~mm})
$$

The time series of angles of deflection $\theta_{1}(t)$ and $\theta_{2}(t)$ were taken for a range of Mach numbers from 0.3 to 0.9 for two test-section lengths. Time series and a power spectrum for the upstream beam deflection for $L=160 \mathrm{~cm}$ for $M=0.9$ are presented in Figures 4(a) and 4(b), respectively. Note the presence of several peaks at low frequencies below $5 \mathrm{kHz}$. These are due to tunnel mechanical vibrations. The modulus and the argument of the spectral cross-correlation function $S(\omega)$, Eq. (10), are plotted in Figures 5(a) and 5(b). The best least- square fit to compute the time delay given by Eq. (5) is represented by the solid line in Figure 5(b).

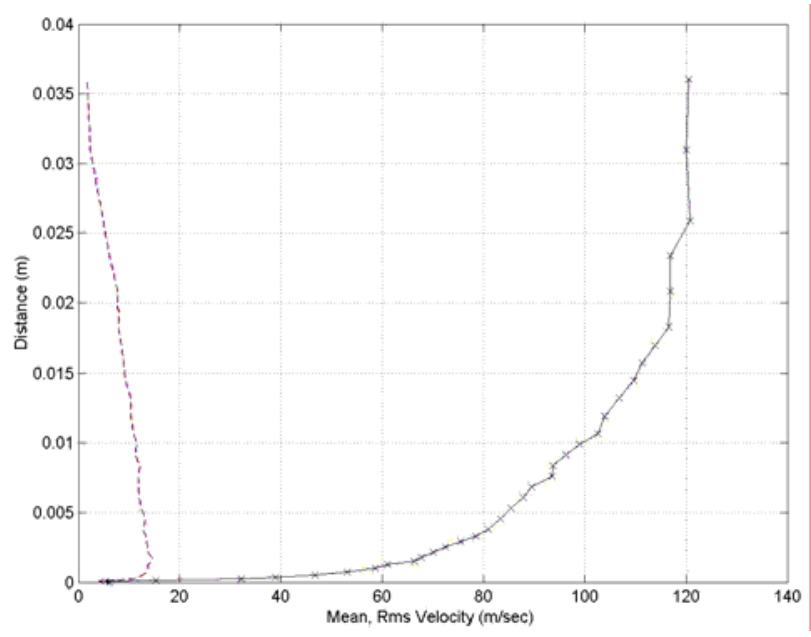

Figure 3. Boundary layer profile at $M=0.38$ for $L=160 \mathrm{~cm}$.

The presence of low-frequency peaks with zerocorrelation phase can be clearly seen in Fig. 5(b) around $4 \mathrm{kHz}$. In order to remove these vibration effects, the data records were filtered using the technique described in Refs. ${ }^{8,9}$. The delay times $\tau^{\max }$ and convective speeds $U_{c}$ were computed from the two filtered signals using both techniques described in the Aberration Convective Speed Determination section above. An example of a time-correlation function $R(\tau)$ is shown in Fig. 6 . Typical levels of maximum correlations were observed to be around 0.5. Results from both methods agree with each other quite well; however, the spectral technique is more robust in cases of low-frequency noise contamination because it relies on phase information at the high frequencies. On the other hand, the time-domain technique can give significant errors if the level of lowfrequency noise (i.e. vibration) is high. Knowing the convective speed, $\mathrm{OPD}_{\text {rms }}$ can be calculated. The results are summarized in Table 2.

The convective speed of structures was found to be roughly 0.8 of the free-stream speed. $O P D_{\text {rms }}$ were on the order of 0.01 to $0.06 \mu \mathrm{m}$ and go up with increasing Mach number. Also, the thicker the boundary layer, i.e., the larger $L$, the higher the optical distortions. The OPD variations can be plotted as a function of a pseudospatial coordinate $x$ assuming the convective nature of distortions, i.e., $t \rightarrow x=-U_{c} t$. For example, the results for $M=0.9$ and $L=160 \mathrm{~cm}$ are presented in Fig. 7; note a presence of large-scale structures in the OPD, on the order of boundary layer thickness. Also an intermittent behavior of the OPD, with periods of high peak-to-peak values, is also evident in Fig. 7. In a later 


\begin{tabular}{|c|c|c|}
\hline$L=110 \mathrm{~cm}$ & $M$ & $\delta^{*}(\mathrm{~mm})$ \\
\hline & 0.40 & 3.77 \\
\hline & 0.55 & 2.65 \\
\hline
\end{tabular}

\begin{tabular}{|c|c|c|}
\hline$L=160 \mathrm{~cm}$ & $M$ & $\delta^{*}(\mathrm{~mm})$ \\
\hline & 0.38 & 4.50 \\
\hline & 0.52 & 4.13 \\
\hline
\end{tabular}

Table 1. Boundary layer displacement thicknesses for different Mach numbers.

section, it will be shown that this intermittent behavior can produce periodic drop outs in far-field intensity patterns, which could be critical to free-space communication links.
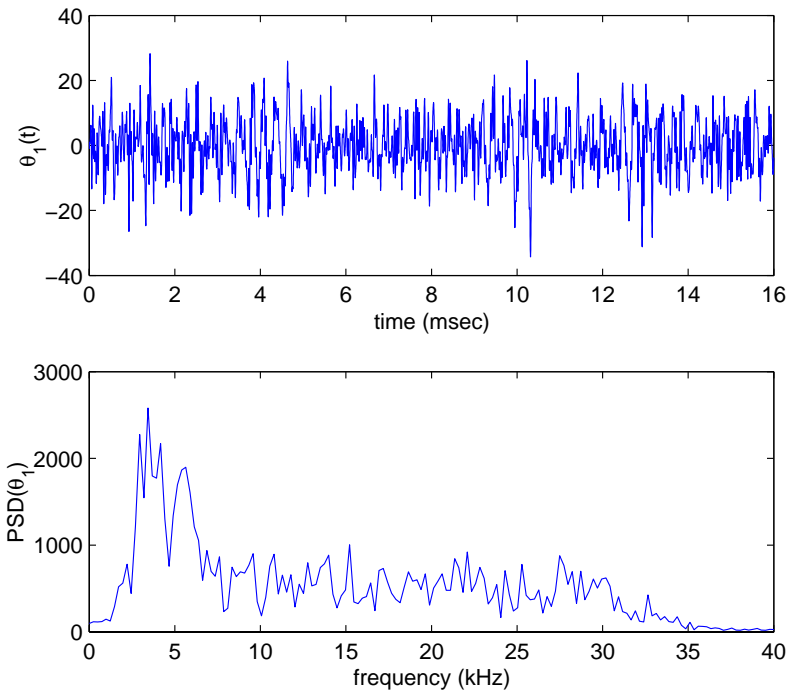

Figure 4. Time series and power spectrum of the beam deflection $\theta_{1}(t)$.

Optical Distortion of Vortical Structures. In order to better understand the nature of the disturbances, a simple model for optical aberrations in a turbulent boundary layer, based on the assumption that periodic, large-scale (order of the boundary-layer thickness) vortices are present in the intermittent region of the boundary layer, was developed. If these are present, then, in the same way that the coherent vortical structures in a free shear layer lead to low-pressure regions in the layer, ${ }^{2}$ a theory was developed to estimate the required pressure gradients that must be present to counter the centrifugal forces created by a

\begin{tabular}{|l|l|l|l|}
\multicolumn{5}{c}{$L=110 \mathrm{~cm}$} \\
\hline$M$ & $U_{\infty}$ & $U_{c}$ & $\mathrm{OPD}_{\mathrm{rms}}(\mu \mathrm{m})$ \\
\hline 0.42 & 142 & 115 & 0.010 \\
\hline 0.55 & 185 & 155 & 0.018 \\
\hline 0.73 & 241 & 199 & 0.034 \\
\hline 0.93 & 298 & 251 & 0.043 \\
\hline
\end{tabular}

\begin{tabular}{|l|l|l|l|}
\multicolumn{5}{c|}{$L=160 \mathrm{~cm}$} \\
\hline $\mathrm{M}$ & $U_{\infty}$ & $U_{c}$ & $\mathrm{OPD}_{\text {rms }}(\mu \mathrm{m})$ \\
\hline 0.54 & 181 & 151 & 0.020 \\
\hline 0.71 & 234 & 185 & 0.036 \\
\hline 0.92 & 294 & 245 & 0.052 \\
\hline
\end{tabular}

Table 2. Convective speeds and $O P D_{\text {rms }}$ data for different boundary layers.
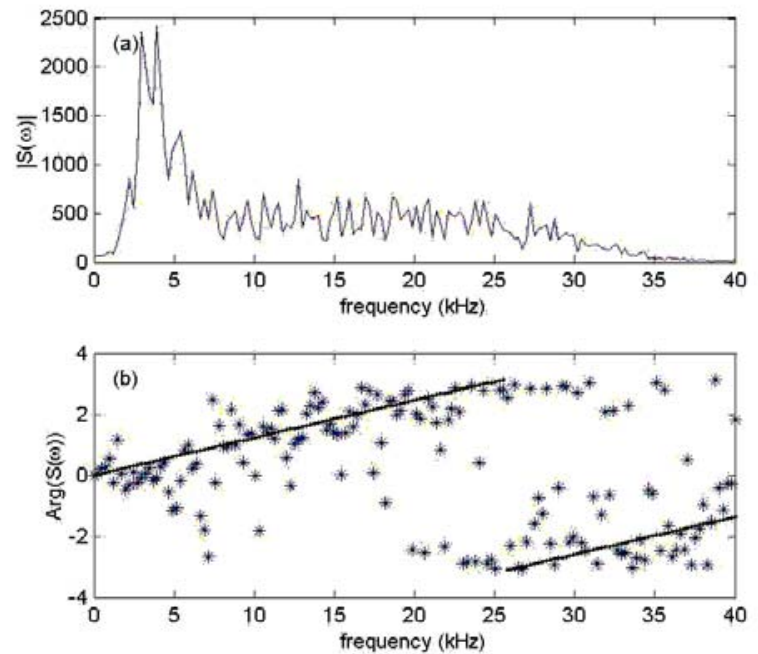

Figure 5. Cross-spectral function $S(\omega)$ : (a) amplitude and (b) phase.

curvature of the flow present in the vortices. Under this assumption, the pressure field is not constant inside of the vortical structure; these fluctuating pressure fields, in turn, create non-uniform density, and thus index-ofrefraction distributions.

Consider a free, two-dimensional vortex with a finite core size, whose azimuthal velocity profile is given by

$$
u_{\theta}(r)=2 u_{\theta}^{\max }\left(r / r_{0}\right) /\left(1+\left(r / r_{0}\right)^{2}\right)
$$

where $r_{0}$ is the vortical core size and $u_{\theta}^{\max }$ is the maximum azimuthal velocity. For a two-dimensional, 
axisymmetrical vortex, the Euler equations reduce to a single equation for a pressure distribution across the vortex

$$
-\frac{u_{\theta}^{2}}{r}=-\frac{1}{\rho} \frac{\partial p}{\partial r}
$$

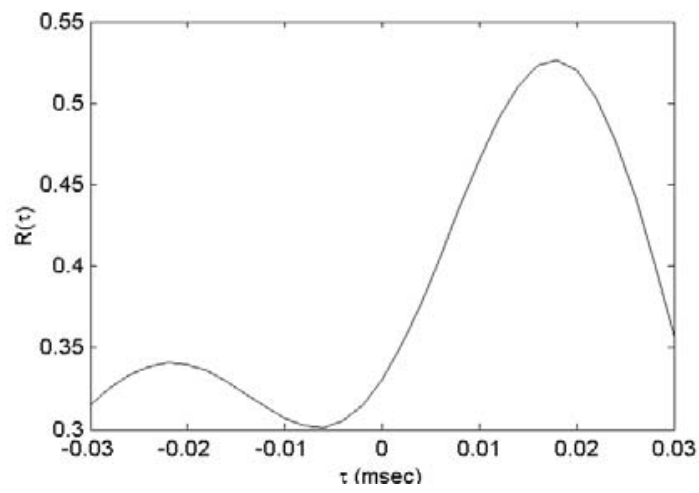

Figure 6. Time-delayed correlation function
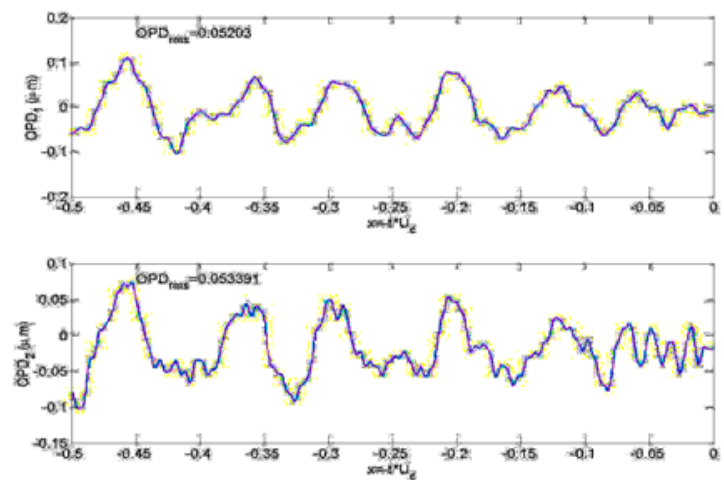

Figure 7. Pseudo-spatial variation of the OPD.

Assuming an isentropic relation between the density and the pressure, as was done in ${ }^{9}$,

$$
p / p_{0}=\left(\rho / \rho_{0}\right)^{\gamma}
$$

the solution for the density distribution for small density fluctuations $\Delta \rho / \rho_{\infty}<<1$ can be found as follows,

$$
\frac{\rho-\rho_{0}}{\rho_{0}}=-\frac{2}{\gamma}\left(\frac{u_{\theta}^{\max }}{a_{\infty}}\right)^{2} \frac{1}{1+\left(r / r_{0}\right)^{2}}
$$

Knowing the density variations as a function of space, the OPL can then be calculated directly by integrating the index of refraction along the propagation path as

$$
O P L(x)=\int n(x) d s=\int n_{0}\left(1+K_{G D}\left(\rho-\rho_{0}\right) / n_{0}\right) d s
$$

where $K_{G D}=0.000227 \mathrm{~m}^{3} / \mathrm{kg}$ is the Gladstone-Dale constant. For propagation directly through the center line of the vortex, i.e., substituting Eq. (22) into Eq. (24) and integrating along $r$ from $-\infty$ to $+\infty$, the OPL is

$$
O P L_{\text {vortex }}=O P L_{\text {free-space }}-2 \pi / \gamma\left(u_{\theta}^{\max } / a_{\infty}\right)^{2} K_{G D} \rho_{0} r_{0}(25)
$$

Thus, the maximum OPD induced by the vortex is

$$
O P L_{\text {peak-to-peak }}=2 \pi / \gamma\left(u_{\theta}^{\max } / a_{\infty}\right)^{2} K_{G D} \rho_{0} r_{0}
$$

Now consider a continuous line of vortices moving with a constant speed. Each vortex would distort a stationary beam and produce a time-varying OPD with a peak-topeak value given by Eq. (26). If these disturbances produce an near sinusoidal aberration, then $O P D_{\text {rms }}$ $\approx 1 / 2.8 O P D_{\text {peak-to-peak. This gives a relationship for }}$ optical distortions as a function of free-stream density $\rho_{0}$, free-stream velocity $U_{\infty}$, vortex intensity $u_{\theta}^{\max }$ and vortex size $r_{0}$,

$$
\begin{aligned}
& O P D_{r m s} \approx \frac{2 \pi}{2.8 \gamma} K_{G D}\left(u_{\theta}^{\max } / a_{\infty}\right)^{2} \rho_{0} r_{0} \\
& \text { or } \quad O P D_{r m s} \approx 3.2 K_{G D} t^{2} M_{\infty}^{2} \rho_{0} r_{0},
\end{aligned}
$$

where $M_{\infty}=U_{\infty} / a_{\infty}$, if $t=\frac{1}{\sqrt{2}} u_{\theta}^{\max } / U_{\infty}$ can be treated as a relative rms turbulence intensity.

Notice that Eq. (27) gives a linear dependence of optical aberration on the dynamic pressure, consistent with the free shear layer weakly-compressible model ${ }^{9}$ and ${ }^{14}$, but not consistent with Gilbert ${ }^{5}$, who suggest a square root of dynamic pressure dependence.

Scaling for the Turbulent Boundary Layer Data. Using scaling arguments described in the previous section, the boundary data can be re-plotted as a function of $M^{2} \rho_{0} / \rho_{S L} \delta^{*}$, where $\delta^{*}$ is a displacement boundary layer thickness and $\rho_{S L}$ is a reference sealevel density. Based on the measured boundary-layer properties, it appeared that the boundary layer was fully turbulent; in the fully-turbulent case, the relative turbulent intensity, $t$, is constant. Our data showed that a typical value for the maximum relative turbulent intensity was $t=7 \%$, see Fig. 3 . The measured $\mathrm{OPD}_{\text {rms }}$ results plotted against the scaling suggested from Eq. (27) are shown in Figure 8. As can be seen in Fig. 8, all the data collapsed on a single straight line. The leastsquare fit analysis gives the following empirical 
expression for $O P D_{\text {rms }}$, represented by a dashed line in Figure 8,

$$
O P D_{r m s}=2.4 \times 10^{-5} \delta^{*} \rho_{0} / \rho_{S L} M^{2}
$$

This expression can be highly useful for interpreting experimental data, since it gives OPD dependence as a function of freestream Mach number, boundary-layer thickness and altitude. For instance, at sea-level $\left(\rho_{0}=\rho_{\mathrm{SL}}\right.$ ) for $M=0.9$ and $\delta^{*}=5 \mathrm{~mm}$, Eq. (28) predicts $O P D_{\text {rms }}=0.079 \mu \mathrm{m}$. The Strehl ratio computed using the large aperture approximation, Eq.(2), for an IR laser, with $\lambda=1 \mu \mathrm{m}$, to be $S_{t}=0.78$. It is interesting to note that if $r_{o}$ in Eq. (27) is taken to be approximately $1 / 2$ the boundary-layer thickness, $\delta$ (for a turbulent boundary layer $\frac{\delta}{\delta^{*}} \approx 6$ ), and the value of the Gladstone Dale constant and the measured turbulence intensity are substituted into Eq. (27) the resulting, simple-model scaling would give

$$
O P D_{r m s}=1.1 \times 10^{-5} \delta \rho_{0} / \rho_{S L} M^{2}
$$

The Figure-8 scaling can be used to estimate the expected system impact by computing the timeaveraged Strehl ratio using Eq. (2); however, because we actually have time histories of $\operatorname{OPD}(t)$ at the measurement location, If we assume that the aberration convects relatively unchanged, it is possible to construct a time series of $\operatorname{OPD}(x, t)$ over by using the

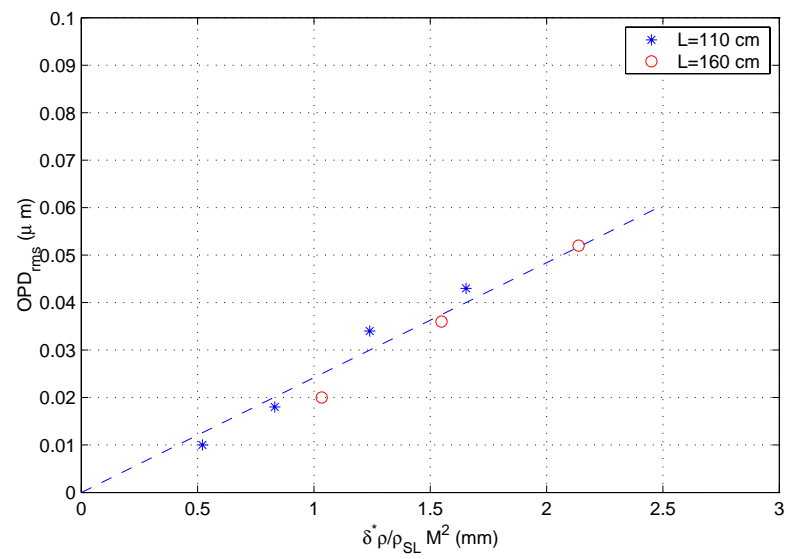

Figure 8. $O P D_{\text {rms }}$ scaling.

convective velocity to trade the time dependence of the OPD at the measurement location into spatial descriptions over an aperture of diameter, $\mathrm{D}$, centered at the measurement location. Given time series of OPD's over an aperture, Fourier optics can used to compute the time-resolved Strehl ratio as a function of time. This was done for three aperture sizes, $D=2,5$ and $20 \mathrm{~cm}$, with the measured OPD rescaled for a sea level; the results for $M=0.9$ are plotted in Fig. 9 for two laser wavelengths, $0.63 \mu \mathrm{m}$ and $1.0 \mu \mathrm{m}$. As can be seen from Fig. 9, significant deviations from the large aperture approximation (marked by the solid straight line in the figure) can be observed. In particular, the intermittent nature of the OPD over the aperture, occasionally causes a large OPD to convect through the aperture, causing dramatic drops in the Strehl ratio for a durations on the order of a millisecond. While the average Strehl ratio is still quite high, these dramatic dropouts can be critical for free-space communication, since they would result in optical-link interruptions and unacceptable bit error rates.
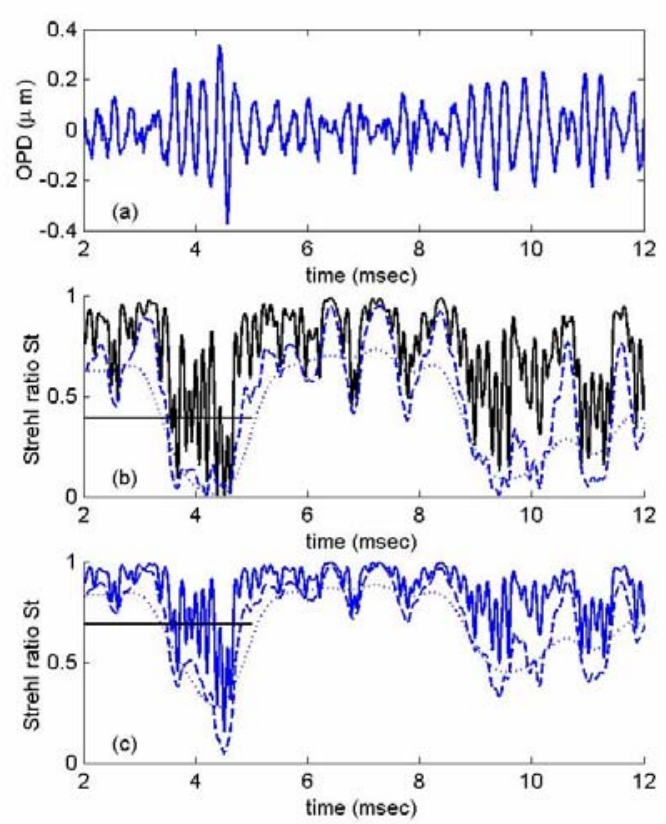

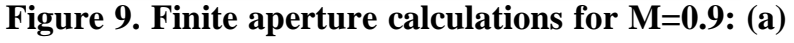
OPD time series, adjusted for a sea-level density; (b) Strehl ratio temporal variation for $\lambda=0.63 \mu \mathrm{m}$, (c) Strehl ratio temporal variation for $\lambda=1.0 \mu \mathrm{m}$. Beam diameters $D=2,5$ and $20 \mathrm{~cm}$ are represented by solid, dashed and dotted lines, respectively. The solid straight line marks the large-aperture limit.

\section{CONCLUSIONS}

The present effort made use of a new measurement technique, the Malley probe, to make a detailed study of the aero-optic characteristics of attached, turbulent, high-subsonic-Mach-number boundary layers. The global results show the prior conclusion that the attached boundary layer is optically more benign than its free-shear-layer counterpart, as suggested in earlier studies, still holds; however, it should be pointed out 
that any impression about the negligible system impact of a turbulent boundary layer that may be left from the earlier implications to $10.6 \mathrm{~mm}$ radiation, should be reassessed by their effect for shorter, near-visible wavelengths, even using the large-aperture approximation given by Eq. (2). With the additional information available about the actual character of the OPD using the Malley probe, it is now possible to compute timeresolved far-field patterns, from which the time dependence of the Strehl ratio can be computed, which was done here. These new detailed time histories show that the attached turbulent boundary layer can cause large excursions in Strehl ratio from that inferred from the large-aperture. These excursions can cause much larger system implications than had previously been assumed. The periodic dropouts, for example, could have devastating affects on attempts to use the beam for a free-space communication link.

The present study has also produced a remarkable scaling relation for the $\mathrm{OPD}_{\mathrm{rms}}$ as a function of Mach number, altitude, and boundary layer thickness. This scaling when properly re-written, is consistent with previously-published empirical findings, $\operatorname{Rose}^{10}$. On the other hand, the scaling suggested by Gilbert $^{5}$ does not appear to be consistent with our findings.

It should be noted that this scaling was initially derived using the simple model presented here. What is even more remarkable than the fact that the theoretical scaling provided a beautiful fit to the data is that the actual constant obtained from the model, Eq. (29), is so close to the experimentally-determined constant, Eq. (28). While the underlying physical cause of the optical aberrations in a turbulent boundary layer, as described in the derivation of the simple model, is by no means proven by these coincidences, they are at least suggestive that this may, in fact, be the underlying cause of the aberrations. Other indirect evidence that the suggested cause could be correct was also found, such as the large "structure size" of the OPD and their high convective speeds; both of these point toward structures in the outer part of the boundary layer. These too seem to support the suppositions contained in our simple model.

On the other hand, the idea that there are largescale coherent vortical structures in the intermittency region of the boundary layer seem counterintuitive, since the most energetic structures (i.e., regions of high vorticity) are confined to the inner part of the boundary layer near the wall. Having said that, it should be noted that our simple model would not prevent the presence of smaller active structures near the wall, since optical aberrations are proportional not only to how "energetic" the structures are, but, even more importantly, to their size. Thus the model might permit the presence of energetic inner structures, but because of their size they are optically less disturbing, while the structures in the outer portion of the boundary layer, being larger in scale albeit perhaps/probably weaker, dominate the overall optical distortion. In order to fully understand the nature of the boundary-layer structures (large and small), additional correlation measurements of the timeresolved velocity inside the boundary layer and the concomitant optical disturbances must be further investigated.

\section{ACKNOWLEDGEMENT}

This work was supported through an AFOSR STTR contract, contract number F49620-02-C-0051. This effort was conducted under the program management of Dr. Thomas Beutner.

\section{References}

[1] Gilbert, K.G., "Overview of Aero-Optics,” AeroOptical Phenomena, Eds. K.G. Gilbert and L.J. Otten, Vol. 80, Progress in Astronautics and Aeronautics, AIAA, New York, 1982, pp. 1-9.

[2] Jumper, E.J., and E.J. Fitzgerald, "Recent Advances in Aero-Optics," Progress in Aerospace Sciences, 37, 2001, pp.299-339.

[3] Hugo, R.J. and E.J. Jumper, "Applicability of the Aero-Optic Linking Equation to a Highly Coherent, Transitional Shear Layer," Applied Optics, 39 (24), August 2000, pp. 4392-4401.

[4] Cicchiello, J.M., and E.J. Jumper, "Far-Field Optical Degradation due to Near-Field Transmission Through a Turbulent Heated Jet," Applied Optics, 36 (25), pp. 6441-6452, September 1997.

[5] Gilbert, K.G., "KC-135 Aero-Optical BoundaryLayer/Shear-Layer Experiments,” Aero-Optical Phenomena, Eds. K.G. Gilbert and L.J. Otten, Vol. 80, Progress in Astronautics and Aeronautics, AIAA, New York, 1982, pp. 306-324.

[6] Masson, B., J. Wissler, and L. McMackin, “AeroOptical Study of a NC-135 Fuselage Boundary Layer,” AIAA Paper 94-0277, January 1994.

[7] Hugo, R.J., E.J. Jumper, G. Havener, and S.A. Stepanek, "Time-Resolved Wavefront Measurements through a Compressible Free Shear Layer," AIAA Journal, 35 (4), April 1997, pp. 671677. 
[8] Fitzgerald, E.J. and E.J. Jumper, “Aperture Effects on the Aero-Optical Distortions Produced by a Compressible Shear layer”, AIAA Journal, 40(2), 2002, pp. 267-275.

[9] Fitzgerald, E.J. and E.J. Jumper, "Further Consideration of Compressibility Effects on ShearLayer Optical Distortion,” AIAA Paper 99-3617, June 1999.

[10] Rose, W.C., "Measurements of Aerodynamic Parameters Affecting Optical Performance," Air Force Weapons Laboratory Final Report, AFWLTR-78-191, May 1979.

[11] Smith, W.J., Modern Optical Engineering: The Design of Optical Systems, McGraw-Hill, NY, 1966, Chap. 3, pp. 49-71.

[12] Malley, M., Sutton, G.W., and Kincheloe, N., "Beam-Jitter Measurements for Turbulent AeroOptical Path Differences, Applied Optics, Vol 31, 1992, pp. 4440-4443.

[13] Hugo, R.J., and Jumper, E.J., "Quantification of Aero-Optical Phase Distortion Using the SmallAperture Beam Technique,” AIAA Journal, 33(11), 1995, pp. 2151-2157]

[14] E. J. Fitzgerald and E. J. Jumper, “ Scaling AeroOptic Aberrations Produced by HighSubsonic-Mach Shear Layers", AIAA Journal, 40(7), 2002, pp. 1373-1381 